\title{
Diffraction of a Plane Elastic Wave by a Gradient Transversely Isotropic Layer
}

\author{
Anastasiia Anufrieva and Dmitrii Tumakov \\ Institute of Computer Science and Information Technology, Kazan Federal University, Kazan 420008, Russia \\ Correspondence should be addressed to Dmitrii Tumakov; dtumakov@ksu.ru
}

Received 24 February 2013; Accepted 5 September 2013

Academic Editor: Akira Ikuta

Copyright ( 2013 A. Anufrieva and D. Tumakov. This is an open access article distributed under the Creative Commons Attribution License, which permits unrestricted use, distribution, and reproduction in any medium, provided the original work is properly cited.

\begin{abstract}
The problem of diffraction of a plane elastic wave by a gradient transversely isotropic layer is considered. Using the method of overdetermined boundary value problem in combination with the Fourier transform method, the system of ordinary differential equations of the second order with boundary conditions of the third type is obtained which is solved by the grid method. Results of calculations obtained using the above-mentioned technique for the case of piecewise linear profiles for the Young modulus of the layer are given.
\end{abstract}

\section{Introduction}

In nature, many of the geological formations form layered structures with elastic properties differing in various directions. Of all the formations and media, the special interest is often given to transversely isotropic media in which elastic modula of the media are the same in the plane normal to the axis of symmetry but differ from those of the direction along the axis of symmetry. Studies show that many sedimentary rocks indeed are transversely isotropic [1-3]. Besides, a thinlayered packet of parallel beds each of which is isotropic but properties of which differ from properties of the other beds within the packet behaves as a transversely isotropic medium at presence of deformations.

Furthermore, transversely isotropic structures are normally used at production of composites. If fibers packed in parallel are used as a reinforcing agent, then the composite possesses a unidirectional structure and is treated as a transversely isotropic material in the planes normal to the direction of reinforcement [4]. Most often sheet metals are not isotropic and possess normal anisotropy (transversely isotropic). Ferroconcrete containing cracks is considered a transversely isotropic material with the plane of isotropy parallel to the plane of the crack [5]. Transversely isotropic structures also occur at production of laminated wood [6].
A number of works have been dedicated to studying processes of propagation of sound waves through anisotropic elastic layers. For example, in [7, 8], an elastic layer was considered as uniform and anisotropic whereas [9] dealt with the problem of propagation of the sound wave through a transversely isotropic nonuniform layer. A simpler case of the problem was considered by the authors of present paper earlier [10]. In the present work considered is the problem of diffraction of an elastic wave by a nonuniform transversely isotropic plate with constant elasticity characteristics along the axis of the layer and a continuous distribution of elasticity parameters in the section.

Differential equations governing the diffraction problem are considered separately for half-planes and for a plate. The problems for half-planes are overdetermined allowing establishing a relationship between traces of desired functions at the media's interface [11]. Thus, the original problem reduces to the boundary value problem for the Lame system with the boundary conditions of the third type. Then the Fourier transform with respect to the variable for which uniformity is preserved is applied to the boundary value problem. The obtained system of ordinary differential equations is solved using the grid method.

Using the above-mentioned technique, dependence of energy of transmitted wave on angle and frequency of 


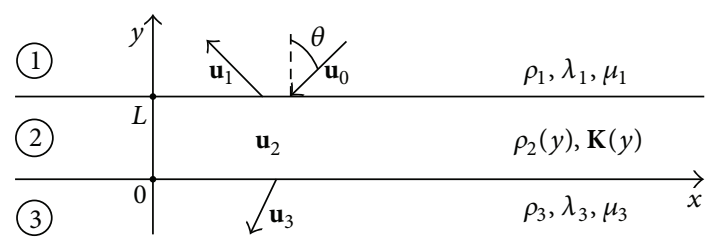

FIgURE 1: Geometry of the problem.

incidence is studied numerically. Differences in behavior of energy of transmitted wave at diffraction by uniformly anisotropic and nonuniformly anisotropic layer are outlined.

\section{Statement of the Problem}

Let an elastic harmonic wave of type $\mathbf{u}_{0}(x, y) \exp \{i \omega t\}$ fall on a nonuniform in the transverse direction anisotropic layer of thickness $L$ (medium $2\{0<y<L\}$ ) with continuous density $\rho_{2}(y)$ and tensor $(3 \times 3)$ of elasticity modulus $\mathbf{K}(y)$ from medium $1\{y>L\}$ under the angle $\theta$ with respect to the axis $y$ (see Figure 1). The diffraction results in a wave $\mathbf{u}_{1}(x, y)$ reflected toward medium 1 , a wave $\mathbf{u}_{3}(x, y)$ propagating toward medium $3\{y<0\}$, and field $\mathbf{u}_{2}(x, y)$ in the layer. Desired is the full diffracted field. Media 1 and 3 are assumed to be uniform and isotropic.

We seek a solution to the plane harmonic problem from the elasticity theory at $y<0$ and $y>L$ in the form

$$
\begin{gathered}
\frac{\partial \sigma_{x n}}{\partial x}+\frac{\partial \tau_{n}}{\partial y}+\rho_{n} \omega^{2} u_{x n}=0, \\
\frac{\partial \tau_{n}}{\partial x}+\frac{\partial \sigma_{y n}}{\partial y}+\rho_{n} \omega^{2} u_{y n}=0, \\
\sigma_{x n}=\left(\lambda_{n}+2 \mu_{n}\right) \frac{\partial u_{x n}}{\partial x}+\lambda_{n} \frac{\partial u_{y n}}{\partial y}, \\
\sigma_{y n}=\lambda_{n} \frac{\partial u_{x n}}{\partial x}+\left(\lambda_{n}+2 \mu_{n}\right) \frac{\partial u_{y n}}{\partial y}, \\
\tau_{n}=\mu_{n}\left(\frac{\partial u_{x n}}{\partial y}+\frac{\partial u_{y n}}{\partial x}\right)
\end{gathered}
$$

for $n=1,3$ with the constant Lame coefficients $\lambda_{n}, \mu_{n}$ and density $\rho_{n}$.

General equations of two-dimensional oscillations are written in the form

$$
\begin{gathered}
-\rho_{2} \omega^{2} u_{x 2}=\frac{\partial \sigma_{x 2}}{\partial x}+\frac{\partial \tau_{2}}{\partial y}, \\
-\rho_{2} \omega^{2} u_{y 2}=\frac{\partial \tau_{2}}{\partial x}+\frac{\partial \sigma_{y 2}}{\partial y}, \\
\sigma_{x 2}=k_{x x x x} \frac{\partial u_{x 2}}{\partial x}+k_{x x x y}\left(\frac{\partial u_{x 2}}{\partial y}+\frac{\partial u_{y 2}}{\partial x}\right)+k_{x x y y} \frac{\partial u_{y 2}}{\partial y},
\end{gathered}
$$

$$
\begin{gathered}
\sigma_{y 2}=k_{y y x x} \frac{\partial u_{x 2}}{\partial x}+k_{y y x y}\left(\frac{\partial u_{x 2}}{\partial y}+\frac{\partial u_{y 2}}{\partial x}\right)+k_{y y y y} \frac{\partial u_{y 2}}{\partial y}, \\
\tau_{2}=k_{x y x x} \frac{\partial u_{x 2}}{\partial x}+k_{x y x y}\left(\frac{\partial u_{x 2}}{\partial y}+\frac{\partial u_{y 2}}{\partial x}\right)+k_{x y y y} \frac{\partial u_{y 2}}{\partial y},
\end{gathered}
$$

where $k_{* * * *}$ are components of the elasticity modulus tensor. Let us introduce a standard notation for indices $k_{* * * *}$ : $\{x x\} \rightarrow\{1\},\{y y\} \rightarrow\{2\}$ and $\{x y\} \rightarrow\{3\}$ and substitute (4) into (3) to obtain

$$
\begin{array}{r}
-\rho_{2} \omega^{2} u_{x 2}=\frac{\partial}{\partial x}\left(k_{11} \frac{\partial u_{x 2}}{\partial x}+k_{13}\left(\frac{\partial u_{x 2}}{\partial y}+\frac{\partial u_{y 2}}{\partial x}\right)\right. \\
\left.+k_{12} \frac{\partial u_{y 2}}{\partial y}\right) \\
+\frac{\partial}{\partial y}\left(k_{13} \frac{\partial u_{x 2}}{\partial x}+k_{33}\left(\frac{\partial u_{x 2}}{\partial y}+\frac{\partial u_{y 2}}{\partial x}\right)\right. \\
\left.+k_{23} \frac{\partial u_{y 2}}{\partial y}\right), \\
-\rho_{2} \omega^{2} u_{y 2}=\frac{\partial}{\partial x}\left(k_{13} \frac{\partial u_{x 2}}{\partial x}+k_{33}\left(\frac{\partial u_{x 2}}{\partial y}+\frac{\partial u_{y 2}}{\partial x}\right)\right. \\
\left.+k_{23} \frac{\partial u_{y 2}}{\partial y}\right) \\
+\frac{\partial}{\partial y}\left(k_{12} \frac{\partial u_{x 2}}{\partial x}+k_{23}\left(\frac{\partial u_{x 2}}{\partial y}+\frac{\partial u_{y 2}}{\partial x}\right)\right. \\
\left.+k_{22} \frac{\partial u_{y 2}}{\partial y}\right) .
\end{array}
$$

We assume that rotational components of the forces can not result in stretching of the body. Then some of the components become equal zero: $k_{13}=k_{23}=0$. Note that, for the case of isotropic body, the elasticity modulus tensor takes the following form:

$$
\mathbf{K}=\left(\begin{array}{ccc}
\lambda+2 \mu & \lambda & 0 \\
\lambda & \lambda+2 \mu & 0 \\
0 & 0 & \mu
\end{array}\right)
$$

At the media's interface, the following conjugation conditions are to be fulfilled:

$$
\begin{gathered}
u_{x 1}(x, L+0)+u_{x 0}(x, L+0)=u_{x 2}(x, L-0), \\
u_{y 1}(x, L+0)+u_{y 0}(x, L+0)=u_{y 2}(x, L-0), \\
\tau_{1}(x, L+0)+\tau_{0}(x, L+0)=\tau_{2}(x, L-0), \\
\sigma_{y 1}(x, L+0)+\sigma_{y 0}(x, L+0)=\sigma_{y 2}(x, L-0),
\end{gathered}
$$


at $y=L$ and

$$
\begin{gathered}
u_{x 3}(x, 0-0)=u_{x 2}(x, 0+0), \\
u_{y 3}(x, 0-0)=u_{y 2}(x, 0+0), \\
\tau_{3}(x, 0-0)=\tau_{2}(x, 0+0), \\
\sigma_{y 3}(x, 0-0)=\sigma_{y 2}(x, 0+0),
\end{gathered}
$$

at $y=0$.

Of all the possible solutions to the system (1), (2), (5), (7), and (8), we pick solutions corresponding to the waves going to infinity.

\section{Boundary Value Problem for the System of Ordinary Differential Equations Describing a Field in the Gradient Layer}

Desired functions for the Lame system (5) for any fixed $y$ from the interval $(0, L)$ will be considered in the class $L_{1, \text { loc }}$, and it will be assumed that the functions undergo a slow growth at infinity in the $x$-direction. This allows applying the Fourier transform with respect to the $x$-direction permitting both waves decaying at infinity and propagating waves. Thus, we perform the change of variables from variable $x$ to variable $\xi$ and obtain the system of equations at $y \in(0, L)$ :

$$
\begin{aligned}
&\left(k_{33} u_{x 2}^{\prime}\right)^{\prime}+\left[\rho_{2} \omega^{2}-k_{11} \xi^{2}\right] u_{x 2} \\
&-i \xi\left(k_{12}+k_{33}\right) u_{y 2}^{\prime}-i \xi k_{33}^{\prime} u_{y 2}=0 \\
&\left(k_{22} u_{y 2}^{\prime}\right)^{\prime}+\left[\rho_{2} \omega^{2}-k_{33} \xi^{2}\right] u_{y 2} \\
&-i \xi\left(k_{12}+k_{33}\right) u_{x 2}^{\prime}-i \xi k_{12}^{\prime} u_{x 2}=0
\end{aligned}
$$

with respect to the Fourier transform for displacements $u_{x 2}(\xi, y)$ and $u_{y 2}(\xi, y)$.

It is worth noting here that the unknowns $u_{x 2}(\xi, y)$ and $u_{y 2}(\xi, y)$ with respect to $y$ are ordinary functions, and, therefore, all the derivatives are to be understood in the classical sense. This allows discretization of the problem with respect to $y$. For any fixed $y$, desired functions with respect to $\xi$ are distributions of the slow growth.

For the upper half-plane $\{y>L\}$, it will be assumed that the solutions $(1),(2)$ belong to $L_{1, \text { loc }}(R)$ and their traces $\tau_{1}(x, L+0), \sigma_{y 1}(x, L+0), u_{x 1}(x, L+0)$ and $u_{y 1}(x, L+0)$ are correctly specified. We will consider that the desired functions are distributions of the slow growth at infinity, and, moreover, their traces are also distributions of the slow growth at infinity. In the work [11], it was shown that solutions corresponding to the wave moving in the positive $y$-direction satisfy the equalities linking to each other Fourier transforms of traces of components of the field

$$
\begin{aligned}
\xi \tau_{1}(\xi, L) & +\gamma_{1,1}(\xi) \sigma_{y 1}(\xi, L) \\
& -2 i \mu_{1} \xi \gamma_{1,1}(\xi) u_{x 1}(\xi, L) \\
& -i\left(\rho_{1} \omega^{2}-2 \mu_{1} \xi^{2}\right) u_{y 1}(\xi, L)=0
\end{aligned}
$$

$$
\begin{aligned}
-\gamma_{2,1}(\xi) & \tau_{1}(\xi, L)+\xi \sigma_{y 1}(\xi, L) \\
+ & i\left(\rho_{1} \omega^{2}-2 \mu_{1} \xi^{2}\right) u_{x 1}(\xi, L) \\
& -2 i \mu_{1} \xi \gamma_{2,1}(\xi) u_{y 1}(\xi, L)=0,
\end{aligned}
$$

where $k_{11}^{2}=\rho_{1} \omega^{2} /\left(\lambda_{1}+2 \mu_{1}\right), k_{21}^{2}=\rho_{1} \omega^{2} / \mu_{1}$ and branches of roots of the functions $\gamma_{1,1}=\sqrt{k_{11}^{2}-\xi^{2}}, \gamma_{2,1}=\sqrt{k_{21}^{2}-\xi^{2}}$ are chosen such that the real part is positive, and in the case of the real part being zero, positive imaginary roots are chosen.

In equalities (10), traces of all desired functions are considered at $y=L$, but since the considered functions are continuous in the whole domain, the limit will be considered as the value at $y=L$. We will proceed with the other traces of the desired functions in the same manner.

We perform transition from traces of functions of medium 1 to traces of functions of the layer in equalities (10). For doing this, we express the traces via conditions (7) and substitute the obtained expressions into (10). Thus, we obtain the following boundary conditions for the Fourier transforms of components of the field defined in the layer

$$
\begin{aligned}
\xi \tau_{2}(\xi, L) & +\gamma_{1,1}(\xi) \sigma_{y 2}(\xi, L) \\
& -2 i \mu_{1} \xi \gamma_{1,1}(\xi) u_{x 2}(\xi, L) \\
& -i\left(\rho_{1} \omega^{2}-2 \mu_{1} \xi^{2}\right) u_{y 2}(\xi, L)=f_{1}(\xi) \\
-\gamma_{2,1}(\xi) & \tau_{2}(\xi, L)+\xi \sigma_{y 2}(\xi, L) \\
+ & i\left(\rho_{1} \omega^{2}-2 \mu_{1} \xi^{2}\right) u_{x 2}(\xi, L) \\
& -2 i \mu_{1} \xi \gamma_{2,1}(\xi) u_{y 2}(\xi, L)=f_{2}(\xi),
\end{aligned}
$$

where

$$
\begin{aligned}
f_{1}(\xi)= & \xi \tau_{0}(\xi, L)+\gamma_{1,1}(\xi) \sigma_{y 0}(\xi, L) \\
& -2 i \mu_{1} \xi \gamma_{1,1}(\xi) u_{x 0}(\xi, L) \\
& -i\left(\rho_{1} \omega^{2}-2 \mu_{1} \xi^{2}\right) u_{y 0}(\xi, L), \\
f_{2}(\xi)= & -\gamma_{2,1}(\xi) \tau_{0}(\xi, L) \\
& +\xi \sigma_{y 0}(\xi, L)+i\left(\rho_{1} \omega^{2}-2 \mu_{1} \xi^{2}\right) u_{x 0}(\xi, L) \\
& -2 i \mu_{1} \xi \gamma_{2,1}(\xi) u_{y 0}(\xi, L) .
\end{aligned}
$$

We eliminate Fourier transforms of the stresses from the obtained conditions using (4). Thus, we obtain relations between traces of Fourier transforms of displacements in the layer

$$
\begin{array}{r}
a_{1}(\xi) u_{x 2}^{\prime}(\xi, L)+a_{2}(\xi) u_{x 2}(\xi, L) \\
+a_{3}(\xi) u_{y 2}^{\prime}(\xi, L) \\
+a_{4}(\xi) u_{y 2}(\xi, L)=f_{1}(\xi), \\
a_{5}(\xi) u_{x 2}^{\prime}(\xi, L)+a_{6}(\xi) u_{x 2}(\xi, L) \\
+a_{7}(\xi) u_{y 2}^{\prime}(\xi, L) \\
+a_{8}(\xi) u_{y 2}(\xi, L)=f_{2}(\xi),
\end{array}
$$


where

$$
\begin{gathered}
a_{1}(\xi)=k_{33}(L), \\
a_{2}(\xi)=-\left(k_{12}(L)+2 \mu_{1}\right) i \xi \gamma_{1,1}(\xi), \\
a_{3}(\xi)=k_{22}(L) \gamma_{1,1}(\xi), \\
a_{4}(\xi)=-i\left(\rho_{1} \omega^{2}-\left(2 \mu_{1}-k_{33}(L)\right) \xi^{2}\right), \\
a_{5}(\xi)=-k_{33}(L) \gamma_{2,1}(\xi), \\
a_{6}(\xi)=i\left(\rho_{1} \omega^{2}-\left(k_{12}(L)+2 \mu_{1}\right) \xi^{2}\right), \\
a_{7}(\xi)=k_{22}(L) \xi, \\
a_{8}(\xi)=-i\left(2 \mu_{1}-k_{33}(L)\right) \xi \gamma_{2,1}(\xi) .
\end{gathered}
$$

On the lower half-plane $\{y<0\}$, solutions (1) will be sought in the class $L_{1, \text { loc }}$ with the slow growth at infinity taking into account that the traces $\tau_{3}(x, 0), \sigma_{y 3}(x, 0), u_{x 3}(x, 0)$, and $u_{y 3}(x, 0)$ are correctly determined and they also belong to $L_{1, \text { loc }}$. Then solutions from the class of distributions of the slow growth corresponding to waves moving in the negative $y$-direction satisfy equations establishing relations between Fourier transforms of components of the field [11]

$$
\begin{aligned}
& \xi_{3}(\xi, 0)-\gamma_{1,3}(\xi) \sigma_{y 3}(\xi, 0) \\
&+2 i \mu_{3} \xi \gamma_{1,3}(\xi, 0) u_{x 3}(\xi) \\
&-i\left(\rho_{3} \omega^{2}-2 \mu_{3} \xi^{2}\right) u_{y 3}(\xi, 0)=0 \\
& \gamma_{2,3}(\xi) \tau_{3}(\xi, 0)+\xi \sigma_{y 3}(\xi, 0) \\
&+i\left(\rho_{3} \omega^{2}-2 \mu_{3} \xi^{2}\right) u_{x 3}(\xi, 0) \\
&+2 i \mu_{3} \xi \gamma_{2,3}(\xi) u_{y 3}(\xi, 0)=0
\end{aligned}
$$

which are equivalent to conditions related to traces of the field components at the lower boundary

$$
\begin{aligned}
& \xi \tau_{2}(\xi, 0)-\gamma_{1,3}(\xi) \sigma_{y 2}(\xi, 0) \\
&+2 i \mu_{3} \xi \gamma_{1,3}(\xi) u_{x 2}(\xi, 0) \\
&-i\left(\rho_{3} \omega^{2}-2 \mu_{3} \xi^{2}\right) u_{y 2}(\xi, 0)=0, \\
& \gamma_{2,3}(\xi) \tau_{2}(\xi, 0)+\xi \sigma_{y 2}(\xi, 0) \\
&+i\left(\rho_{3} \omega^{2}-2 \mu_{3} \xi^{2}\right) u_{x 2}(\xi, 0) \\
&+2 i \mu_{3} \xi \gamma_{2,3}(\xi) u_{y 2}(\xi, 0)=0
\end{aligned}
$$

where $k_{13}^{2}=\rho_{3} \omega^{2} /\left(\lambda_{3}+2 \mu_{3}\right), k_{23}^{2}=\rho_{3} \omega^{2} / \mu_{3}$ and branches of roots of the functions $\gamma_{1,3}=\sqrt{k_{13}^{2}-\xi^{2}}, \gamma_{2,3}=\sqrt{k_{23}^{2}-\xi^{2}}$ are chosen in the same way as branches of roots of the functions of the upper half-plane.
Using (4), we obtain

$$
\begin{gathered}
b_{1}(\xi) u_{x 2}^{\prime}(\xi, 0)+b_{2}(\xi) u_{x 2}(\xi, 0) \\
+b_{3}(\xi) u_{y 2}^{\prime}(\xi, 0) \\
+b_{4}(\xi) u_{y 2}(\xi, 0)=0 \\
b_{5}(\xi) u_{x 2}^{\prime}(\xi, 0)+b_{6}(\xi) u_{x 2}(\xi, 0) \\
+b_{7}(\xi) u_{y 2}^{\prime}(\xi, 0) \\
+b_{8}(\xi) u_{y 2}(\xi, 0)=0
\end{gathered}
$$

where

$$
\begin{gathered}
b_{1}(\xi)=k_{33}(0) \xi \\
b_{2}(\xi)=i\left(k_{12}(0)+2 \mu_{3}\right) \xi \gamma_{1,3}(\xi), \\
b_{3}(\xi)=-k_{22}(0) \gamma_{1,3}(\xi), \\
b_{4}(\xi)=-i\left(\rho_{3} \omega^{2}-\left(2 \mu_{3}-k_{33}(0)\right) \xi^{2}\right), \\
b_{5}(\xi)=k_{33}(0) \gamma_{2,3}(\xi), \\
b_{6}(\xi)=i\left(\rho_{3} \omega^{2}-\left(k_{12}(0)+2 \mu_{3}\right) \xi^{2}\right), \\
b_{7}(\xi)=k_{22}(0) \xi \\
b_{8}(\xi)=i\left(2 \mu_{3}-k_{33}(0)\right) \xi \gamma_{2,3}(\xi) .
\end{gathered}
$$

Physical meanings of solutions of (9) with the boundary conditions (13) and (17) are displacements $\left(u_{x 2}, u_{y 2}\right)$ which describe the field at $0<y<L$ in the problem of diffraction in the elastic layer.

\section{Elastic Oscillations of a Transversely Isotropic Body}

Let us consider three-dimensional oscillations of an elastic transversely isotropic medium. To describe deformations of the medium, the following model will be used [12]:

$$
\begin{gathered}
\varepsilon_{x x}=\frac{1}{E}\left(\sigma_{x x}-v \sigma_{z z}\right)-\frac{\nu^{\prime}}{E^{\prime}} \sigma_{y y}, \\
\varepsilon_{z z}=\frac{1}{E}\left(\sigma_{z z}-v \sigma_{x x}\right)-\frac{\nu^{\prime}}{E^{\prime}} \sigma_{y y}, \\
\varepsilon_{y y}=-\frac{\nu^{\prime}}{E^{\prime}}\left(\sigma_{x x}+\sigma_{z z}\right)+\frac{1}{E^{\prime}} \sigma_{y y}, \\
\varepsilon_{x y}=\frac{1}{2 G^{\prime}} \sigma_{x y}, \quad \varepsilon_{x z}=\frac{1}{2 G} \sigma_{x z}, \\
\varepsilon_{y z}=\frac{1}{2 G^{\prime}} \sigma_{y z} .
\end{gathered}
$$

Here the plane $x z$ is the plane of isotropy and the planes $x y$ and $y z$ are the planes of elastic symmetry. The parameters $E, E^{\prime}$ are the Young modula, $v$ and $v^{\prime}$ are the Poisson coefficients, $G=E /(2(1+\nu))$ and $G^{\prime}$ are the displacement 
modula. Parameters without the prime sign correspond to deformations in the plane of isotropy whereas parameters with the prime sign correspond to deformations in the plane of elastic symmetry.

Equations (19) could be transformed to the form [13]

$$
\begin{gathered}
\sigma_{x x}=(\lambda+2 \mu) \varepsilon_{x x}+\lambda \varepsilon_{z z}+\lambda^{\prime} \varepsilon_{y y}, \\
\sigma_{z z}=\lambda \varepsilon_{x x}+(\lambda+2 \mu) \varepsilon_{z z}+\lambda^{\prime} \varepsilon_{y y}, \\
\sigma_{y y}=\lambda^{\prime}\left(\varepsilon_{x x}+\varepsilon_{z z}\right)+\left(\lambda^{\prime}+2 \mu^{\prime}\right) \varepsilon_{y y}, \\
\sigma_{x y}=2 G^{\prime} \varepsilon_{x y}, \quad \sigma_{x z}=2 \mu \varepsilon_{x z}, \\
\sigma_{y z}=2 G^{\prime} \varepsilon_{y z},
\end{gathered}
$$

in which the used notations imply the following:

$$
\begin{array}{r}
\lambda+2 \mu=\frac{E}{(1+\nu) d}\left(1-\left(\nu^{\prime}\right)^{2} \frac{E}{E^{\prime}}\right), \\
\lambda=\frac{E}{(1+\nu) d}\left(\nu+\left(\nu^{\prime}\right)^{2} \frac{E}{E^{\prime}}\right), \\
\lambda^{\prime}+2 \mu^{\prime}=\frac{E^{\prime}(1-\nu)}{d}, \\
\lambda^{\prime}=\frac{E \nu^{\prime}}{d}, \quad d=1-\nu-2\left(\nu^{\prime}\right)^{2} \frac{E}{E^{\prime}} .
\end{array}
$$

We will assume that the field does not depend on the $z$ coordinate: $\partial / \partial z \equiv 0$. Then we have $\varepsilon_{z z}=0$, and the system of (20) falls into two independent subsystems. The first subsystem describes oscillations in the plane $x y$ :

$$
\begin{gathered}
\sigma_{x x}=(\lambda+2 \mu) \varepsilon_{x x}+\lambda^{\prime} \varepsilon_{y y}, \\
\sigma_{y y}=\lambda^{\prime} \varepsilon_{x x}+\left(\lambda^{\prime}+2 \mu^{\prime}\right) \varepsilon_{y y} \\
\sigma_{x y}=2 G^{\prime} \varepsilon_{x y},
\end{gathered}
$$

whereas the second subsystem describes oscillations in the $z$ direction:

$$
\begin{gathered}
\sigma_{z z}=\lambda \varepsilon_{x x}+\lambda^{\prime} \varepsilon_{y y}, \quad \sigma_{x z}=2 \mu \varepsilon_{x z}, \\
\sigma_{y z}=2 G^{\prime} \varepsilon_{y z} .
\end{gathered}
$$

The system (24) with the use of equations of motion transforms to the following:

$$
\mu \frac{\partial^{2} u_{z}}{\partial x^{2}}+\frac{\partial}{\partial y}\left(G^{\prime} \frac{\partial u_{z}}{\partial y}\right)+\rho_{2} \omega^{2} u_{z}=0
$$

The system (23) corresponds to (4) with the following notations: $\sigma_{x 2}=\sigma_{x x}, \sigma_{y 2}=\sigma_{y}$, and $\tau_{2}=\sigma_{x y}$. Under the conditions $k_{13}=k_{23}=0$ considered in Section 1, the elasticity tensor $\mathbf{K}$ linking stress and deformations to each other takes the following form:

$$
\mathbf{K}=\left(\begin{array}{ccc}
\lambda+2 \mu & \lambda^{\prime} & 0 \\
\lambda^{\prime} & \lambda^{\prime}+2 \mu^{\prime} & 0 \\
0 & 0 & G^{\prime}
\end{array}\right)
$$

Thus, the problem of diffraction of an elastic harmonic wave by a transversely isotropic layer reduces to the boundary value problem (9), (13), and (17) with the elasticity tensor defined in (26).

\section{Numerical Results}

Before discussions of the numerical results, we will give some notes regarding dependence of solution of the problem (9), (13), and (17) on parameter $\xi$. All the coefficients of the boundary value problem are continuous functions of $\xi$. Then if right-hand sides of (13) are regular distributions on $\xi$, then solutions will also be considered as regular with respect to $\xi$. However, if $f_{1}$ and $f_{2}$ are singular distributions on $\xi$, then the solutions themselves will also be considered as singular. For example, if $f_{1}=C_{1} \delta\left(\xi-\xi_{0}\right)$ and $f_{2}=C_{2} \delta\left(\xi-\xi_{0}\right)$, then $u_{x}(y)=\delta\left(\xi-\xi_{0}\right) w_{x}\left(y ; \xi_{0}\right)$ and $u_{y}(y)=\delta\left(\xi-\xi_{0}\right) w_{y}\left(y ; \xi_{0}\right)$. In this case, it is convenient to "normalize" the boundary value problem by $\delta\left(\xi-\xi_{0}\right)$. For doing that, we perform the change of variables from variable $\xi$ to variable $\xi_{0}$ all over and solve (9), (13), and (17) with respect to $w_{x}\left(y ; \xi_{0}\right)$ and $w_{y}\left(y ; \xi_{0}\right)$.

Therefore, in the case of Fourier transforms of traces of the incident field being singular distributions, for example, in the case of the incident wave being a plane wave, the solutions of the problems will also be singular distributions with the same carrier. From this it follows that diffraction of one plane wave results in two reflected waves: longitudinal and transverse, and excitation of waveguide waves in the layer does not occur. It is obvious that the last statement is true under condition of uniqueness of the diffraction problem (homogeneous conditions (13) result in a trivial solution to the problem (9), (13), and (17)) and under condition the eigenvalues of the waveguide formed by the layer which differ from $\xi_{0}$.

The desired problem can be solved using many approximation methods. A uniform, finite-difference grid with the mesh size $h$ was chosen to approximate the boundary value problem (9), (13), and (17) with the accuracy on the order of $O(h)$. When choosing the mesh size it is taken into account that the finite difference analogs of elastic profiles of the layer describe adequately the original continuous models. On the other hand, the mesh size $h$ must be smaller than the wavelength in the layer and, consequently, inversely proportional to the frequency $\omega$.

After carrying out the numerical solution, it is necessary to reconstruct the fields in the half-planes $u_{1}$ and $u_{3}$. For doing it, we consider displacements in a homogeneous isotropic $n$th medium which can be written in the general form in the following way [14]:

$$
\begin{aligned}
u_{x n}(y)= & \xi A_{n} e^{-i \gamma_{1 n} y}-\xi B_{n} e^{i \gamma_{1 n} y} \\
& +\gamma_{2 n} C_{n} e^{-i \gamma_{2 n} y}+\gamma_{2 n} D_{n} e^{i \gamma_{2 n} y} \\
u_{y n}(y)= & \gamma_{1 n} A_{n} e^{-i \gamma_{1 n} y}+\gamma_{1 n} B_{n} e^{i \gamma_{1 n} y} \\
& -\xi C_{n} e^{-i \gamma_{2 n} y}+\xi D_{n} e^{i \gamma_{2 n} y}
\end{aligned}
$$


Taking into account the conditions at infinity, displacements for the reflected field will have the following form:

$$
\begin{aligned}
& u_{x 1}(y)=-\xi_{0} B_{1} e^{i \gamma_{11}(y-L)}+\gamma_{21} D_{1} e^{i \gamma_{21}(y-L)}, \\
& u_{y 1}(y)=\gamma_{11} B_{1} e^{i \gamma_{11}(y-L)}+\xi_{0} D_{1} e^{i \gamma_{21}(y-L)}
\end{aligned}
$$

and for the transmitted wave

$$
\begin{aligned}
& u_{x 3}(y)=\xi_{0} A_{3} e^{-i \gamma_{13} y}+\gamma_{23} C_{3} e^{-i \gamma_{23} y}, \\
& u_{y 3}(y)=\gamma_{13} A_{3} e^{-i \gamma_{13} y}-\xi_{0} C_{3} e^{-i \gamma_{23} y} .
\end{aligned}
$$

The unknown coefficients $B_{1}, D_{1}, A_{3}$, and $C_{3}$ are found via the following expressions:

$$
\begin{array}{rlrl}
B_{1} & =\frac{u_{y 1} \gamma_{21}-u_{x 1} \xi_{0}}{\gamma_{11} \gamma_{21}+\xi_{0}^{2}}, & D_{1}=\frac{u_{x 1} \gamma_{11}+u_{y 1} \xi_{0}}{\gamma_{11} \gamma_{21}+\xi_{0}^{2}}, \\
A_{3}=\frac{u_{y 3} \gamma_{23}+u_{x 3} \xi_{0}}{\gamma_{13} \gamma_{23}+\xi_{0}^{2}}, & C_{3}=\frac{u_{x 3} \gamma_{13}-u_{y 3} \xi_{0}}{\gamma_{13} \gamma_{23}+\xi_{0}^{2}},
\end{array}
$$

where $u_{x n}$ and $u_{y n}$ are traces of displacements of the $n$th medium which are expressed using (7) and (8).

We will consider the case of diffraction by a plane longitudinal wave with displacements of the following kind:

$$
\begin{aligned}
& u_{x 0}(x, y) \\
&= A_{0} k_{1,1} \sin \theta \\
& \quad \times \exp \left\{-i k_{1,1} \sin \theta x-i k_{1,1} \cos \theta(y-L)\right\} \\
& u_{y 0}(x, y) \\
&=A_{0} k_{1,1} \cos \theta \\
& \quad \times \exp \left\{-i k_{1,1} \sin \theta x-i k_{1,1} \cos \theta(y-L)\right\} .
\end{aligned}
$$

We apply Fourier transformation to components of the incident field and arrive at the result that all the components of the field are singular distributions with the multiplier $\delta(\xi-$ $\left.\xi_{0}\right), \xi_{0}=k_{1,1} \sin \theta$. For example, the Fourier transform of the trace $u_{x 0}(x, y)$ at $y=L$ takes the following form:

$$
u_{x 0}(\xi, L)=A_{0} k_{1,1} \sin \theta \delta\left(\xi-k_{1,1} \sin \theta\right) .
$$

Since the right-hand sides of (13) are singular distributions, then it is sufficient to solve the problem (9), (13), and (17) just at the value $\xi=\xi_{0}$.

For carrying out the numerical experiments, we will consider the case when the layer of thickness $L=10 \mathrm{~m}$ made of siltstone is located in sandstone. Parameters of sandstone filling in medium 1 and medium 3 are $\rho=2400 \mathrm{~kg} / \mathrm{m}^{3}, v_{p}=$ $3300 \mathrm{~m} / \mathrm{sec}$, and $v_{s}=2000 \mathrm{~m} / \mathrm{sec}$. The layer is considered anisotropic; we will consider three cases of distributions of elasticity parameters in the layer. A uniform layer has the following parameters [15]: $E=56.8 \mathrm{GPa}, E^{\prime}=62.1 \mathrm{GPa}$, $v=0.29, v^{\prime}=0.26$, and $G^{\prime}=22.9 \mathrm{GPa}$. In the case of "compressed siltstone," all the parameters of the medium remain constant except for the Young modulus $E(y)$ which,

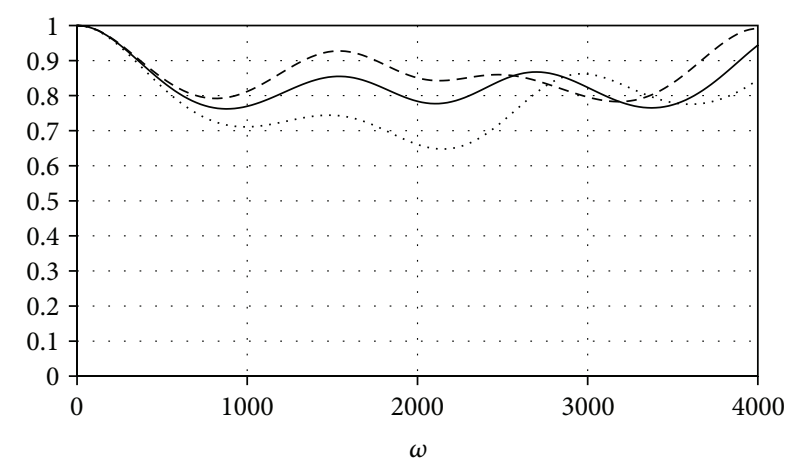

FIGURE 2: Dependence of normalized energy of the transmitted longitudinal wave on angular frequency $\omega$ at its incidence under the angle $\theta=20^{\circ}$ upon a siltstone layer of thickness $L=10 \mathrm{~m}$ placed into sandstone. The solid curve corresponds to a uniform layer of siltstone, the dotted curve "compressed siltstone," and the dashed curve "relaxed siltstone."

in the plane of isotropy, grows linearly from $E(0)=56.8 \mathrm{GPa}$ to $E(L / 2)=85.0 \mathrm{GPa}$ and then reduces linearly to the value $E(L)=56.8 \mathrm{GPa}$. In the case of "relaxed siltstone," the Young modulus $E(y)$ reduces linearly from $E(0)=56.8 \mathrm{GPa}$ to $E(L / 2)=28.4 \mathrm{GPa}$ and grows linearly to $E(L)=56.8 \mathrm{GPa}$.

In the present work, two sets of studies were carried out. The first set of studies is dedicated to searching for dependence of normalized energy of the transmitted longitudinal wave on angular frequency $\omega$. As a result, the conclusion is made so that the transmitted energy grows as the value of the Young modulus $E(y)$ reduces in the middle of the layer. The difference between various structures increases with the increase of angle of incidence $\theta$. The dependences are given in Figures 2 and 3.

In Figure 2, dependence of normalized energy of the transmitted longitudinal wave on angular frequency $\omega$ at its incidence under the angle $\theta=20^{\circ}$ upon a siltstone layer of thickness $L=10 \mathrm{~m}$ placed into sandstone is shown. The solid curve corresponds to a uniform layer of siltstone, the dotted curve "compressed siltstone," and the dashed curve "relaxed siltstone." In Figure 3, the same type of dependence is shown but for the angle $\theta=40^{\circ}$.

The second set of studies is dedicated to searching for dependence of normalized energy of the transmitted longitudinal wave on the angle of incidence $\theta$. Just as in the previous set of studies, confirmed is the conclusion that the transmitted energy increases with the decrease of the Young modulus $E(y)$ in the middle of the layer. It is worth noting here that the difference in the transmitted energy for different structures decreases with the increase of angular frequency $\omega$.

In Figure 4, dependence of normalized energy of the transmitted longitudinal wave on $\theta$ at the angular frequency of the wave $\omega=2 \cdot 10^{3}$ radian per second upon a siltstone layer of thickness $L=10 \mathrm{~m}$ placed into sandstone is shown. The solid curve corresponds to a uniform layer of siltstone, the dotted curve "compressed siltstone," and the dashed curve "relaxed siltstone." In Figure 5, the same type of dependence is shown but for $\omega=4 \cdot 10^{3}$ radian per second. 


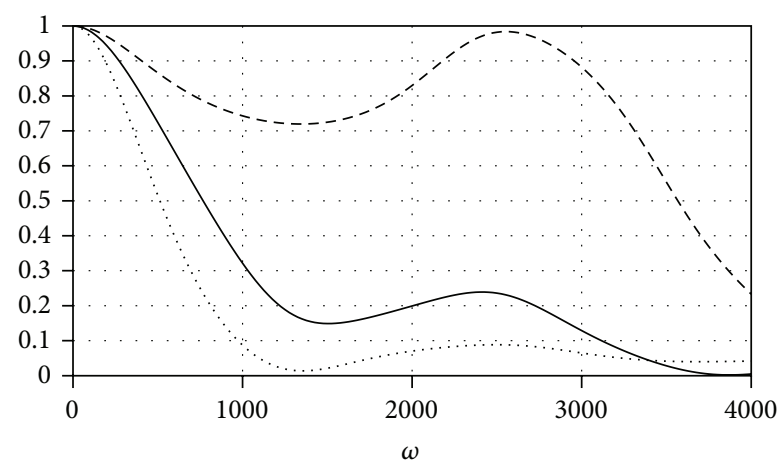

Figure 3: Dependence of normalized energy of the transmitted longitudinal wave on angular frequency $\omega$ at its incidence under the angle $\theta=40^{\circ}$ upon a siltstone layer of thickness $L=10 \mathrm{~m}$ placed into sandstone. The solid curve corresponds to a uniform layer of siltstone, the dotted curve "compressed siltstone," and the dashed curve "relaxed siltstone."

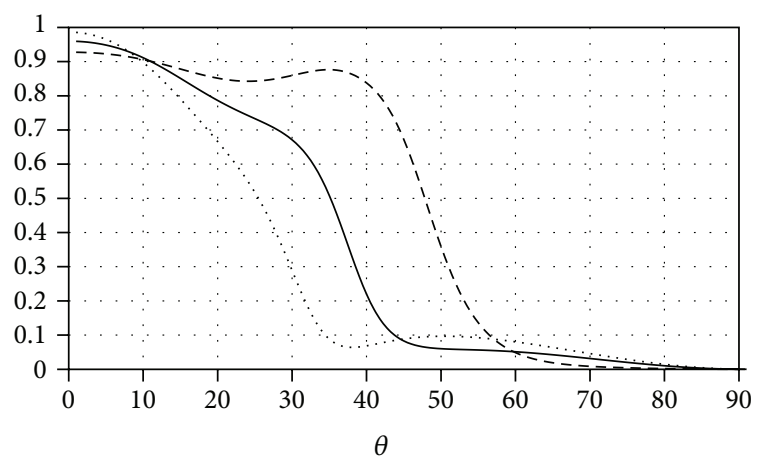

FIGURE 4: Dependence of normalized energy of the transmitted longitudinal wave having the angular frequency $\omega=2000 \mathrm{rad} / \mathrm{sec}$ on its angle of incidence $\theta$ upon a siltstone layer of thickness $L=10 \mathrm{~m}$ placed into sandstone. The solid curve corresponds to a uniform layer of siltstone, the dotted curve "compressed siltstone," and the dashed curve "relaxed siltstone."

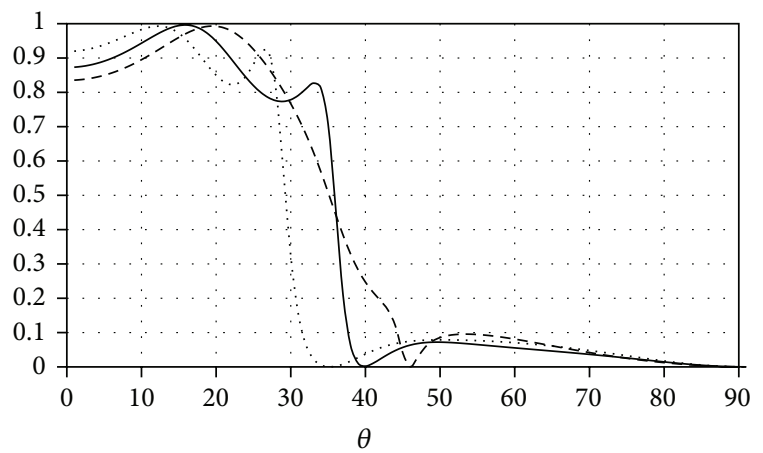

FIGURE 5: Dependence of normalized energy of the transmitted longitudinal wave having the angular frequency $\omega=4000 \mathrm{rad} / \mathrm{sec}$ on its angle of incidence $\theta$ upon a siltstone layer of thickness $\mathrm{L}=10 \mathrm{~m}$ placed into sandstone. The solid curve corresponds to a uniform layer of siltstone, the dotted curve "compressed siltstone," and the dashed curve "relaxed siltstone."

\section{Conclusions}

The method of overdetermined boundary value problem, used in the present work, when combined with and the Fourier transform method is shown to be efficient, especially, for the cases the Fourier transforms of traces of the incident field are singular distributions. Then the approximation problem is solved just at the value $\xi=\xi_{0}$. In the case of the Fourier transforms being regular distributions (e.g., at diffraction of a Gauss beam by a plate), the problem (9), (13), and (17) is solved for several values of the parameter $\xi$.

Results obtained with respect to propagation of elastic waves through anisotropic layers can be used in geophysics for the initial analysis of structure of the layers of rock strata. Also results of propagation of elastic waves through nonuniform anisotropic structures can be used in industries in which anisotropic materials are applied as well as at designing protective layers for various processes and apparatuses.

\section{Acknowledgment}

This work was supported by RFBR 12-01-97012-r-povolzh'e-a.

\section{References}

[1] S. Ryan-Grigor, "Empirical relationships between transverse isotropy parameters and VplVS: implications for AVO," Geophysics, vol. 62, no. 5, pp. 1359-1364, 1997.

[2] T. Alkhalifah, "Velocity analysis using nonhyperbolic moveout in transversely isotropic media," Geophysics, vol. 62, no. 6, pp. 1839-1854, 1997.

[3] C. M. Sayers, "Simplified anisotropy parameters for transversely isotropic sedimentary rocks," Geophysics, vol. 60, pp. 1933-1935, 1995.

[4] V. J. Nemirovskii and A. P. Jankovskii, "Determining effective physical and mechanical characteristics of hybrid composites crisscross reinforced by transversely isotropic fibers and comparisons of computed characteristics versus experimental data," MekhanIka Kompozicionnykh MaterIalov i Konstrukcii, vol. 13, no. 1, pp. 3-32, 2007.

[5] G. A. Geniev, V. N. Kissjuk, and G. A. Tjupin, Theory of Plasticity of Concrete and Ferroconcrete, Strojizdat, Moscow, Russia, 1974.

[6] B. V. Labudin, "Justifying a computational model treating laminated wood as an orthogonal transversely isotropic material," Izvestiya VUZov Lesnoj Zhurnal, no. 6, pp. 136-139, 2006.

[7] M. P. Lonkevich, "Propagation of sound through a layer of a transversely isotropic material of finite thickness," Akusticheskij Zhurnal, vol. 17, no. 1, pp. 85-92., 1971.

[8] E. L. Shenderov, "Propagation of sound through a layer of a transversely isotropic plate," Akusticheskii Zhurnal, vol. 30, no. 1, pp. 122-129, 1984.

[9] S. A. Skobelitsyn and L. A. Tolokonnikov, "Propagation of sound through a transversely isotropic nonuniform flat layer," Akusticheskii Zhurnal, vol. 36, no. 4, pp. 740-744, 1990.

[10] A. V. Anufrieva, D. N. Tumakov, and V. L. Kipot, "Elastic wave propagation through a layer with graded-index distribution of density," in Proceedings of the Days on Diffraction (DD '12), pp. 21-26, 2012.

[11] I. E. Pleshchinskaya and N. B. Pleshchinskii, "Over-determined boundary value problems for linear equations of elastodynamics and their applications to elastic wave diffraction theory," in 
Advances in Mathematics Research, A. R. Baswell, Ed., vol. 17, pp. 102-138, Nova Science, New York, NY, USA, 2012.

[12] S. G. Lekhnickii, Theory of Elasticity of the Anisotropic Body, Nauka, Moscow, Russia, 1977.

[13] B. D. Annin, "Transversely isotropic elastic model of geological materials," Sibirskiı Zhurnal Industrial noй Matematiki, vol. 12, no. 3, pp. 5-14, 2009.

[14] K. N. Vdovina, N. B. Pleshchinskii, and D. N. Tumakov, "Concerning orthogonality of proper waves of a half-opened elastic waveguide," Izvestiya VUZov Matematika, no. 9, pp. 6975, 2008.

[15] S. A. Batugin and R. K. Nirenburg, "Approximate relation between the elastic constants of anisotropic rocks and the anisotropy parameters," Fiziko-Tekhnicheskie Problemy Razrabotki Poleznykh Iskopaemykh, vol. 7, no. 1, pp. 7-12, 1972. 

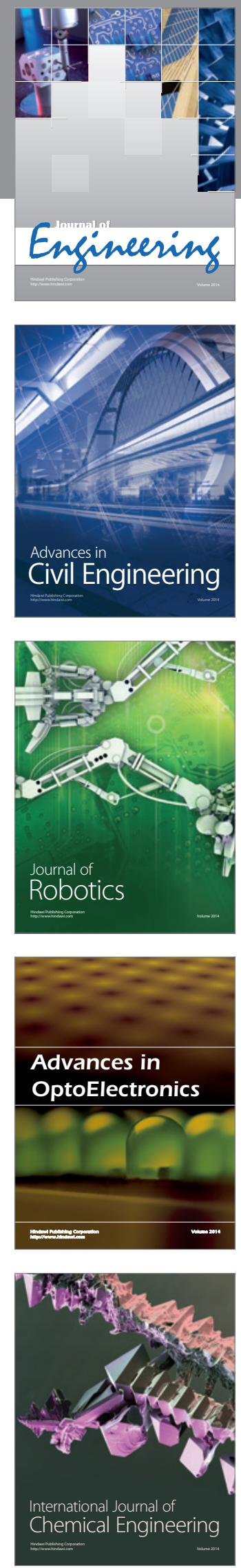

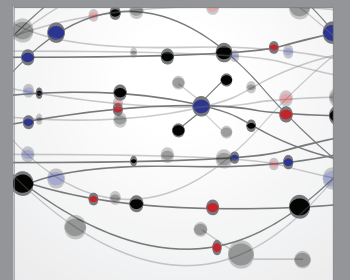

The Scientific World Journal
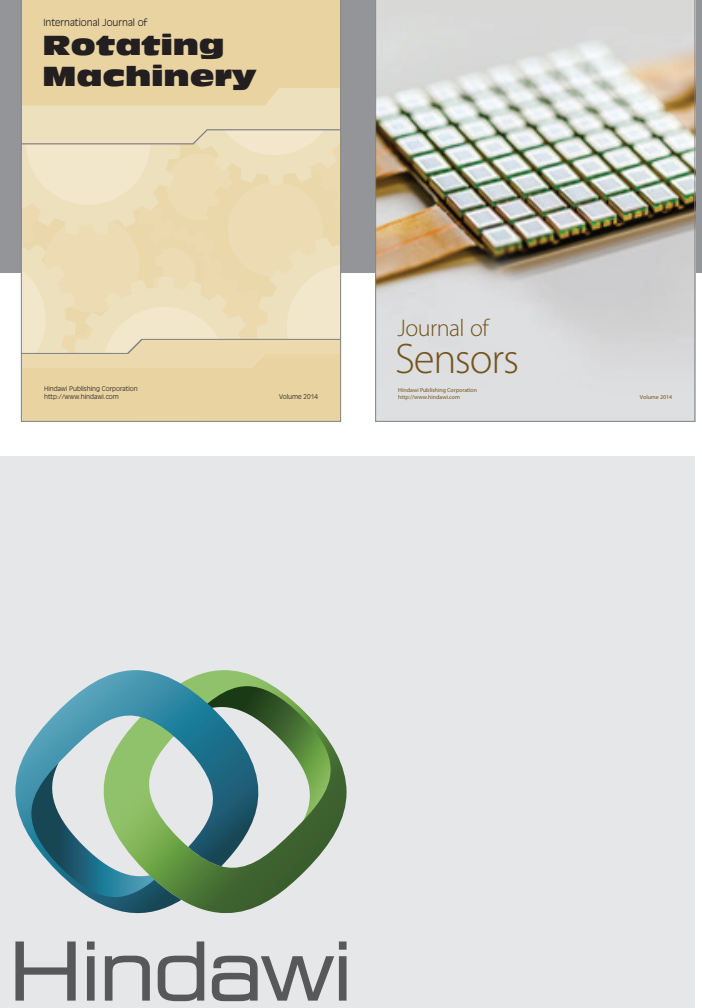

Submit your manuscripts at http://www.hindawi.com
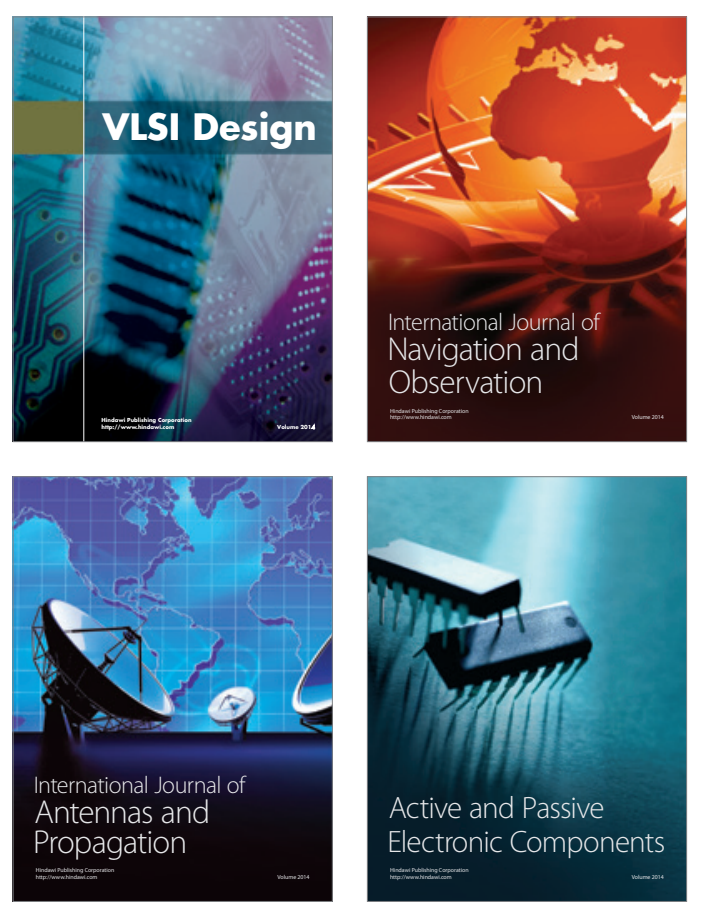
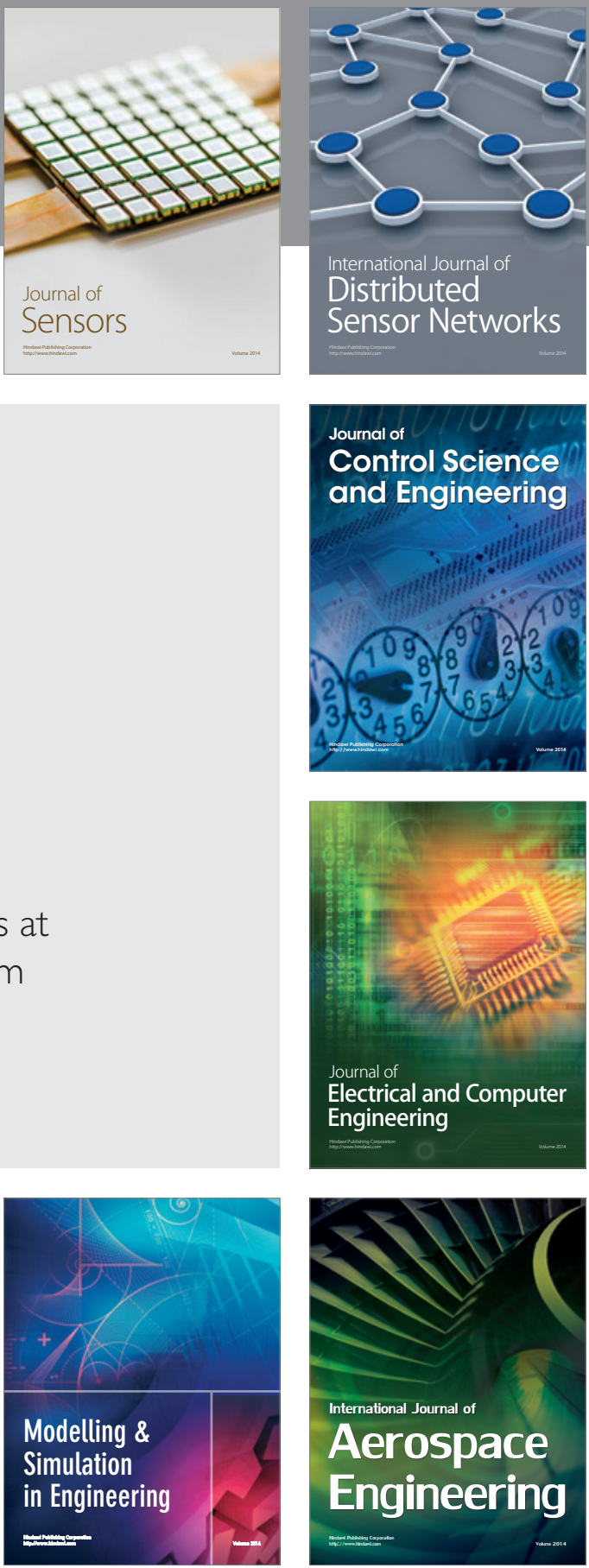

Journal of

Control Science

and Engineering
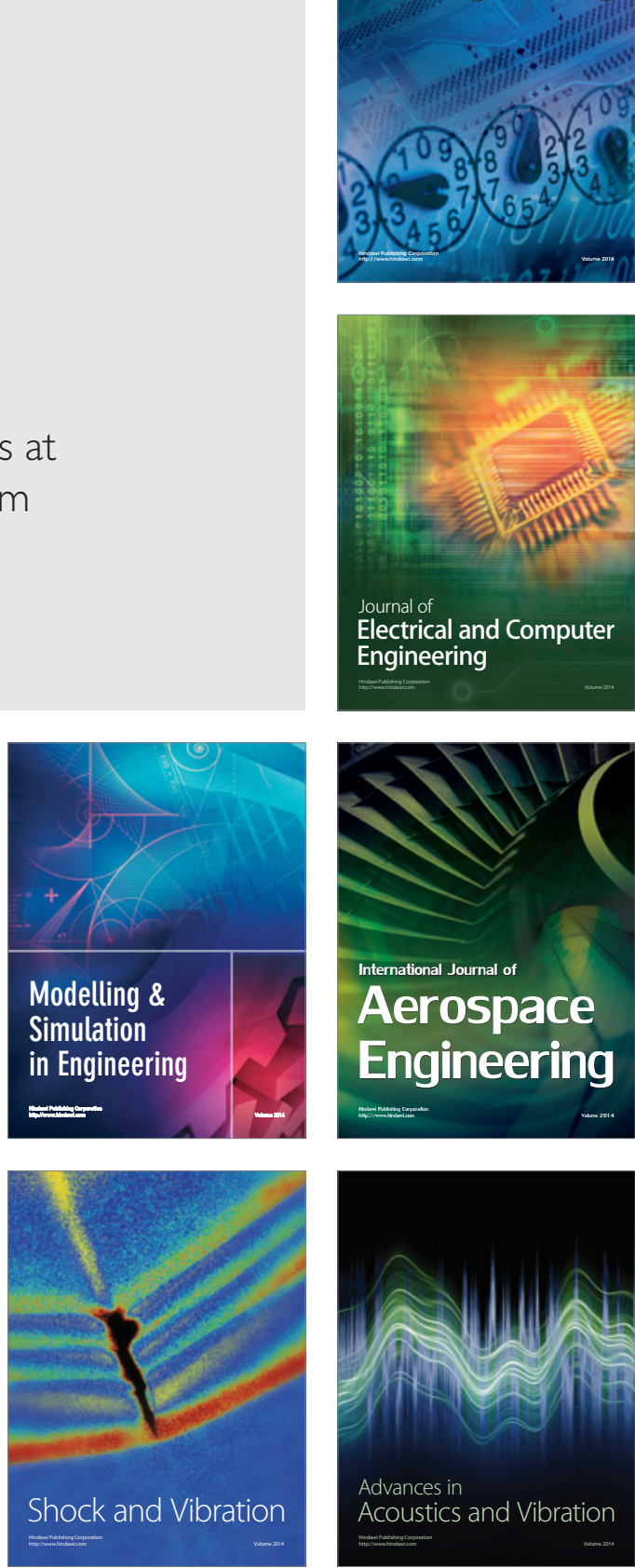